\title{
DNA methylation biomarker for cumulative lead exposure is associated with Parkinson's disease
}

\author{
Kimberly C. Paul ${ }^{1 *} \mathbb{D}$, Steve Horvath², |rish Del Rosario ${ }^{3}$, Jeff M. Bronstein ${ }^{1}$ and Beate Ritz ${ }^{1,3}$
}

\begin{abstract}
Lead, a known neurotoxicant, has previously received attention in Parkinson's disease (PD) research, but epidemiologic studies have been limited in sample size and findings are equivocal. We generated two methylation-based biomarkers for cumulative tibia and patella bone-measured lead exposure in 1528 PD patients and 1169 controls. PD status was associated with increased levels of the DNAm biomarker for tibia-lead levels. We estimated a meta-OR for PD of 1.89 per unit DNAm tibia-lead increase $(95 \% \mathrm{Cl} 1.59,2.24 ; \mathrm{p}=8.1 \mathrm{E}-13)$. The current study supports the notion that chronic and long-term lead exposure tracked via DNAm may contribute to PD pathogenesis.
\end{abstract}

Keywords: Parkinson's Disease, Lead, DNA methylation

\section{Introduction}

Parkinson's disease (PD) is a progressive neurodegenerative disorder characterized by gradual loss of dopaminergic neurons in the substantia nigra. PD etiology is complex and multi-factorial, likely involving a combination of genetic, aging, and environmental components. Lead, a known neurotoxicant, has previously received attention in PD, but findings in epidemiologic studies have been equivocal.

Lead passes through the blood-brain barrier and enters neurons and glia through calcium channels, affecting neurotransmitter release, energy metabolism, and inducing reactive oxygen species (1). Long-term, cumulative lead levels, which depend on both exposure levels and an individual's uptake, storage, and clearance, may affect the aging brain the most. Interestingly, in epidemiologic studies, results for PD association seem to depend on the type of exposure assessment employed. Specifically, studies using bone-lead measurements found positive associations $(2,3)$, while those relying on selfreport of occupational exposures or emissions data, with one exception (4), did not (5-8). Suggesting that errors in exposure measurement and issues of timing may obscure the role lead plays in neurodegeneration.

Clearly, bone-lead-measurement is the gold-standard for long-term lead exposure, given that the half-life of lead in the tibia is approximately 20 years (9). However, cost and feasibility have often precluded this option, especially in larger studies. Recent advances in highthroughput epigenetic technology are paving the way for biomarker discovery in many fields, including exposure science. Two methylation-based biomarkers for cumulative lead exposure measured in the tibia and patella have recently been developed (10). Using these biomarkers, for the first time we evaluate the influence of methylation-based cumulative lead exposure on PD risk in two, independent population-based studies with over 2600 participants. original author(s) and the source, provide a link to the Creative Commons licence, and indicate if changes were made. The images or other third party material in this article are included in the article's Creative Commons licence, unless indicated otherwise in a credit line to the material. If material is not included in the article's Creative Commons licence and your intended use is not permitted by statutory regulation or exceeds the permitted use, you will need to obtain permission directly from the copyright holder. To view a copy of this licence, visit http://creativecommons.org/licenses/by/4.0/. The Creative Commons Public Domain Dedication waiver (http://creativeco mmons.org/publicdomain/zero/1.0/) applies to the data made available in this article, unless otherwise stated in a credit line to the data. 


\section{Methods}

For our analysis, we used genome-wide DNA methylation (DNAm) data from two publicly available PD studies (Gene Expression Omnibus (GEO) accession numbers GSE145361 (SGPD), GSE72774 and GSE72776 (PEG)). DNAm was measured with the Human Methylation $450 \mathrm{k}$ BeadChip in whole blood samples.

The System Genomics of Parkinson's Disease (SGPD) is a consortium of three studies from across Australia and New Zealand (11). The data available on GEO data consists of 1889 samples (959 PD patients and 930 controls) of European ancestry. Prevalent PD patients were recruited (PD duration 2-40+years), and controls consisted primarily of community-based age-matched volunteers from the same communities, as well as some patient's spouses and siblings. Cohort details, DNA extraction methods, quality control procedures, and normalization methods (quantile-normalized and normalization adjusted for batch, slide, cohort, sentrix row/ column, sex, and age) have been described (11).

The Parkinson's Environment and Genes (PEG) study is population-based study from three agricultural counties of Central California (12). GEO data is available for 807 samples (569 PD patients and 238 controls) of European and Hispanic ancestry. Patients early in disease (mean PD duration $=2.9$ years $(\mathrm{SD}=2.3)$ ) were diagnosed in-person by UCLA Movement Disorder Specialists (J.B.). Population-based controls from the same communities were randomly sampled from Medicare lists and via residential tax assessor's records. Cohort details, DNA extraction methods, quality control procedures, and normalization methods have been previously described (13).

We generated two epigenetic biomarkers for cumulative lead exposure (tibia and patella), developed in the
Normative Aging Study (NAS). The epigenetic biosensors of patella and tibia are linear combinations of 59 and $138 \mathrm{CpGs,}$ respectively, identified with site-by-site analysis and combined via machine-learning algorithms trained on $\mathrm{K} \mathrm{x}$-ray fluorescence (KXRF) in-vivo measures of bone-lead (10). To determine lead biomarker levels in SGPD and PEG, we extracted the published regression coefficients from NAS (10), and applied them to the corresponding DNAm beta matrices. At time of publication, the specificity of the DNAm biosensors has not been validated beyond the initial study.

In order to quantify the relationship between the DNAm lead and PD, we used logistic regression to estimate odds ratios (ORs) and 95\% CIs for PD. The OR is a measure of association that compares the odds of $\mathrm{PD}$ based on exposure, with an OR greater than 1 indicating that the odds of PD increase with exposure. Regression diagnostics showed that model assumptions were met. Based on data availability from GEO, we controlled for age (estimated with the Horvath DNAmAge in SGPD (14)), sex, ancestry (PEG only), blood cell composition, smoking history (PEG only), and mean methylation by sample to account for global methylation. We imputed blood cell proportions using the Houseman method (15). We assessed between-study heterogeneity for SGPD and PEG results with Cochran's $Q$, and calculated a meta OR using a fixed-effects model with weights based on precision.

\section{Results}

Characteristics of SGPD and PEG participants can be found in Table 1. In both studies, PD patients were a few years older than their respective controls and more often men. In PEG, there were less former/current smokers

Table 1 Study descriptives

\begin{tabular}{|c|c|c|c|c|}
\hline \multirow[t]{2}{*}{ Demographic variable } & \multicolumn{2}{|l|}{ SGPD } & \multicolumn{2}{|l|}{ PEG } \\
\hline & $P D(n=959)$ & Control $(n=930)$ & $P D(n=569)$ & Control $(n=238)$ \\
\hline \multicolumn{5}{|l|}{ Continuous variables: mean (SD) } \\
\hline $\mathrm{Age}^{*}$ & $66.5(9.1)$ & $64.9(10.4)$ & $70.5(9.8)$ & $67.5(12.8)$ \\
\hline European fractional ancestry & NA & & $0.87(0.27)$ & $0.92(0.18)$ \\
\hline meanMethBySample & $0.26(0.01)$ & $0.27(0.01)$ & $0.29(0.01)$ & $0.29(0.01)$ \\
\hline DNAm tibia-lead & $2.57(0.41)$ & $2.46(0.49)$ & $2.65(0.54)$ & $2.52(0.52)$ \\
\hline DNAm patella-lead & $3.41(0.4)$ & $3.48(0.4)$ & $3.06(0.4)$ & $3.03(0.3)$ \\
\hline \multicolumn{5}{|l|}{ Categorical variables: n (\%) } \\
\hline Male & $611(63.7)$ & $428(46.0)$ & $356(62.6)$ & $127(53.4)$ \\
\hline Former smoker & NA & & $240(42.4)$ & $125(52.5)$ \\
\hline Current smoker & & & $25(4.2)$ & $17(7.1)$ \\
\hline
\end{tabular}

*For SGPD, age was not provided in the GEO data. To account for age, we therefore generated the epigenetic age based on the Horvath epigenetic clock (DNAmAge, highly correlated with chronologic age $\mathrm{R}>0.9$ in other studies of older adults)

NA: All participants with SGPD methylation data are of European ancestry (11). Smoking status data not available for SGPD via data not provided in GEO 
among patients. Additional file 1: Fig. 1 shows box plots of the data, including all data points and the mean values for both DNAm biomarkers by PD status and stratified by study.

PD status was strongly associated with the DNAm biomarker for tibia-lead levels (Table 2) in both cohorts (Cochran's $Q$ indicated no significant heterogeneity study results, $\mathrm{p}=0.25)$. Specifically, with minimal adjustment (age, sex, ancestry), we estimated in SGPD, an OR for PD of 2.06 per unit DNAm tibia-lead increase (95\% CI 1.66, 2.56; $\mathrm{p}=5.4 \mathrm{E}-11)$ and in PEG of 1.60 (95\% CI 1.20, 2.15; $\mathrm{p}=1.0 \mathrm{E}-03$ ), with a meta-OR of 1.89 (95\% CI 1.59, 2.24; $\mathrm{p}=8.1 \mathrm{E}-13)$. Further adjustment for blood cell composition, mean methylation by sample, and smoking status (PEG only), resulted in a meta OR of 1.52 (95\% CI 1.25, 1.86; $\mathrm{p}=2.8 \mathrm{E}-5)$. The DNAm biomarker for patella-lead levels was inversely associated with PD in SGPD (fully adjusted model: $\mathrm{OR}=0.70$ (95\% CI $0.52,0.93)$ ), but not in PEG $(\mathrm{OR}=1.18(95 \% \mathrm{CI} 0.80,1.75)$ (heterogeneity between study results $p=0.006)$. Results for both DNAm markers stratified by sex can be found in Additional file 3: Table 1. Additionally, results were similar in models both with or without adjustment for smoking (Additional file 3: Table 2).

\section{Discussion}

In two large population-based PD studies, we used DNAm to estimate cumulative, bone-lead levels by applying an externally developed epigenetic (DNAm) biosensor (10), and found that PD was strongly associated with the DNAm pattern estimated for tibia-lead levels. Positive associations were seen independently in both studies and persisted after multiple adjustments. Our findings for DNAm tibia-lead are supported by positive associations in two prior epidemiologic studies that relied on actual tibia bone-lead measurements but had more limited sample sizes (121 and 330 PD patients $(2,3)$ ).

We found no association between DNAm patellalead and PD in PEG, and even an inverse-association in the SGPD study. Interestingly, one of the previous epidemiologic studies also only observed tibiabut not patella-lead measurements to be associated with PD (2). This is perhaps due to the half-life of lead, which is approximately 8 years in the patella (representing trabecular bone) versus decades in the tibia (representing cortical bone) $(2,16)$. Thus, tibia-lead measurements represent cumulative exposure from a more distant past than patella. This shorter half-life may also explain why an inverse-association was observed in SGPD, where the patients had a longer PD duration at blood-draw (2-42 years), while PEG patients were enrolled early in disease (mean 2.9 years). Should a primary source of lead exposure among adults be occupationally related, exposure would be less likely to have occurred among patients in the years around PD diagnosis, as patients stop working several years earlier on average than controls due to disease (17). Industries with occupational lead exposure include construction jobs with lead-based paint activities, including removal or demolition, metal and chemical product manufacturing, battery manufacturing and recycling, and electronics manufacturing (18). Other sources of chronic lead exposure, such as drinking water, leaded gasoline, and household paint, however, may be less influenced by PD onset. Overall, multiple sources of evidence from previous and our current study support a positive association for long-term, cumulative lead exposure that extended over many decades, as measured in the tibia, with PD, rather than shorter-term and more recent exposures reflected in patella-lead levels.

The inordinate advantage of our study is the availability of DNAm-lead biomarkers, which also allows for future applications to all PD studies, or more generally studies of age-related disease, with methylation data and largerscale systematic research into the long-term neurotoxicity of lead. The technology and methodology to track many health-related factors through methylation is rapidly developing. However, currently the DNAm-lead biomarkers have two limitations in our opinion, first, that they were developed in an all-male cohort, and second, that the specificity has not yet been validated beyond the

Table 2 DNAm estimated cumulative lead exposure and PD risk

\begin{tabular}{|c|c|c|c|c|c|c|c|c|c|}
\hline & & \multicolumn{2}{|l|}{$\operatorname{SGPD}(n=1889)$} & \multicolumn{2}{|l|}{ PEG $(n=807)$} & \multicolumn{2}{|l|}{ Meta-analysis } & \multicolumn{2}{|c|}{$\begin{array}{l}\text { Test of study } \\
\text { heterogeneity }\end{array}$} \\
\hline & & OR $(95 \% \mathrm{Cl})$ & $p$-value & OR $(95 \% \mathrm{Cl})$ & $p$-value & OR $(95 \% \mathrm{Cl})$ & $p$-value & Q & p-value \\
\hline MODEL 1 & \multirow[t]{2}{*}{ DNAm Tibia-lead } & $2.06(1.66,2.56)$ & $5.4 \mathrm{E}-11$ & $1.60(1.20,2.15)$ & 0.001 & $1.89(1.59,2.24)$ & $8.09 \mathrm{E}-13$ & 1.32 & 0.25 \\
\hline MODEL 2 & & $1.54(1.22,1.95)$ & $2.8 \mathrm{E}-04$ & $1.48(1.03,2.13)$ & 0.019 & $1.52(1.25,1.86)$ & $2.84 \mathrm{E}-05$ & 1.87 & 0.17 \\
\hline MODEL 1 & \multirow[t]{2}{*}{ DNAm Patella-lead } & $0.59(0.45,0.78)$ & $1.6 \mathrm{E}-04$ & $1.19(0.82,1.73)$ & 0.356 & \multirow{2}{*}{\multicolumn{2}{|c|}{$\begin{array}{l}\text { No replication (study heterogene- } \\
\text { ity) }\end{array}$}} & 8.83 & 0.003 \\
\hline MODEL 2 & & $0.70(0.53,0.93)$ & 0.015 & $1.18(0.80,1.75)$ & 0.417 & & & 7.53 & 0.006 \\
\hline
\end{tabular}

Model 1: adjusts for age (DNAmAge in SGPD), sex, ancestry (PEG only)

Model 2: adjusts for age (DNAmAge in SGPD), sex, ancestry (PEG only), smoker (PEG only), blood cell composition, and meanMethBySample 
initial study (10). Future validation of the DNAm-lead biomarkers would be incredibly beneficial to the research community, given the widespread adverse health effects from chronic lead exposure. Our study provides a compelling first glance into the utility of DNAm-lead exposure measures in PD and neurodegenerative research in general, and solid evidence for the involvement of this DNAm-lead exposure pattern in PD pathogenesis.

\section{Supplementary Information}

The online version contains supplementary material available at https://doi. org/10.1186/s13148-021-01051-3.

Additional file 1: Box plot figures of the DNAm lead-biomarkers by PD and stratified by study.

Additional file 2: Table showing DNAm estimated cumulative lead exposure and PD risk, stratified by sex.

Additional file 3: Table showing DNAm estimated cumulative lead exposure and PD risk in PEG, with and without controlling for smoking.

\section{Acknowledgements}

Not applicable.

\section{Authors' contributions}

KCP performed statistical analysis. All authors contributed to data interpretation and writing/editing the manuscript. All authors read and approved the final manuscript.

\section{Funding}

This work was supported by the National Institute of Environmental Health Science (Grant Numbers R21ES024356, 2R01ES010544, U54ES012078).

\section{Availability of data and materials}

The datasets analyzed during the current study are available in the GEO repository, Accession Numbers GSE145361 (SGPD), GSE72774 and GSE72776 (PEG).

\section{Declarations}

\section{Ethics approval and consent to participate}

The PEG study was approved by the UCLA Institutional Review Board (IRB\# 11-001530) and informed consent was obtained from all individuals. Our research conformed to the Declaration of Helsinki.

\section{Consent for publication}

Not applicable, this manuscript does not contain any individual person's data in any form, e.g. individual details, images, or videos.

\section{Competing interests}

The authors declare that they have no competing interests.

\section{Author details}

${ }^{1}$ Department of Neurology, David Geffen School of Medicine, 73-320B CHS Campus, Los Angeles, CA 177220, USA. ${ }^{2}$ Department of Human Genetics, David Geffen School of Medicine, Los Angeles, CA, USA. ${ }^{3}$ Department of Epidemiology, UCLA Fielding School of Public Health, Los Angeles, CA, USA.

\section{References}

1. Reuben A. Childhood Lead Exposure and Adult Neurodegenerative Disease. J Alzheimer's Disease. 2018:64(1):17-42.

2. Weisskopf MG, Weuve J, Nie H, Saint-Hilaire MH, Sudarsky L, Simon DK, et al. Association of cumulative lead exposure with Parkinson's disease. Environ Health Perspect. 2010;118(11):1609-13.

3. Coon S, Stark A, Peterson E, Gloi A, Kortsha G, Pounds J, et al. Wholebody lifetime occupational lead exposure and risk of Parkinson's disease. Environ Health Perspect. 2006;114(12):1872-6.

4. Gorell JM, Johnson CC, Rybicki BA, Peterson EL, Kortsha GX, Brown GG, et al. Occupational exposures to metals as risk factors for Parkinson's disease. Neurology. 1997:48(3):650-8.

5. Seidler A, Hellenbrand W, Robra BP, Vieregge P, Nischan P, Joerg J, et al. Possible environmental, occupational, and other etiologic factors for Parkinson's disease: a case-control study in Germany. Neurology. 1996:46(5):1275-84

6. Firestone JA, Lundin Jl, Powers KM, Smith-Weller T, Franklin GM, Swanson PD, et al. Occupational factors and risk of Parkinson's disease: a population-based case-control study. Am J Ind Med. 2010;53(3):217-23.

7. Palacios N, Fitzgerald K, Roberts AL, Hart JE, Weisskopf MG, Schwarzschild MA, et al. A prospective analysis of airborne metal exposures and risk of Parkinson disease in the nurses' health study cohort. Environ Health Perspect. 2014;122(9):933-8.

8. Willis AW, Evanoff BA, Lian M, Galarza A, Wegrzyn A, Schootman M, et al, Metal emissions and urban incident Parkinson disease: a community health study of medicare beneficiaries by using geographic information systems. Am J Epidemiol. 2010;172(12):1357-63.

9. Hu H, Shih R, Rothenberg S, Schwartz BS. The epidemiology of lead toxicity in adults: Measuring dose and consideration of other methodologic issues. Environ Health Perspect. 2007;115(3):455-62.

10. Colicino E, Just A, Kioumourtzoglou MA, Vokonas P, Cardenas A, Sparrow $D$, et al. Blood DNA methylation biomarkers of cumulative lead exposure in adults. J Expo Sci Environ Epidemiol. 2019;31(1):108-16.

11. Vallerga CL, Zhang F, Fowdar J, McRae AF, Qi T, Nabais MF, et al. Analysis of DNA methylation associates the cystine-glutamate antiporter SLC7A11 with risk of Parkinson's disease. Nat Commun. 2020;

12. Ritz BR, Paul KC, Bronstein JM. Of pesticides and men: a California story of genes and environment in Parkinson's disease. Curr Environ Heal reports. 2016;3(1):40-52.

13. Chuang Y-H, Paul KC, Bronstein JM, Bordelon Y, Horvath S, Ritz B. Parkinson's disease is associated with DNA methylation levels in human blood and saliva. Genome Med. 2017;9(1):76

14. Horvath S. DNA methylation age of human tissues and cell types. Genome Biol. 2013;14(10):96.

15. Houseman EA, Accomando WP, Koestler DC, Christensen BC, Marsit CJ, Nelson $\mathrm{HH}$, et al. DNA methylation arrays as surrogate measures of cell mixture distribution. BMC Bioinformat. 2012;

16. Kim R, Landrigan C, Mossmann P, Sparrow D, Hu H. Age and secular trends in bone lead levels in middle-aged and elderly men: three-year longitudinal follow-up in the normative aging study. Am J Epidemiol. 1997;146(7):586-91.

17. Koerts J, König M, Tucha L, Tucha O. Working capacity of patients with Parkinson's disease - a systematic review. Parkinsonism Relat Disord. 2016;27:9-24

18. Koh DH, Locke SJ, Chen YC, Purdue MP, Friesen MC. Lead exposure in US worksites: A literature review and development of an occupational lead exposure database from the published literature. Am J Ind Med. 2015;58(6):605-16.

\section{Publisher's Note}

Springer Nature remains neutral with regard to jurisdictional claims in published maps and institutional affiliations.

Received: 17 December 2020 Accepted: 7 March 2021

Published online: 22 March 2021 